\title{
Autologous Platelet-Released Growth Factor and Sexual Dysfunction Amendment: A Pilot Clinical Trial of Successful Improvement Sexual Dysfunction after Pelvic Irradiation
}

\author{
Atefeh Samaie Nouroozi ${ }^{1}$, Ashraf Alyasin', Ashraf Malek Mohammadi², Nili \\ Mehrdadi, Seyed Asadollah Mousavi²,3, Mohammad Vaezi ${ }^{2,3}$, Atoosa Gharib ${ }^{4}$, \\ Ardeshir Ghavamzadeh ${ }^{2,3}$, Saeed Mohammadi ${ }^{2,3 *}$
}

\begin{abstract}
Sexual dysfunction (SDF) is a common sequel to cancer treatment which affects the quality of life in women treated with pelvic radiotherapy. The aim of this study was to evaluate the safety, symptom resolution and objective improvement the injection of autologous platelet released growth factor (APRGF) for treatment of SDF in cited patients. This prospective pilot study enrolled 10 cancer-free patients with SDF who underwent pelvic radiotherapy at least 5 years ago, randomly. Each patient was received 1-2 cc APRGF within four weeks and all patients were re-evaluated at eight weeks and six months. CD34 immuno histochemistry and Masson's trichrome staining were performed on vaginal biopsy section for angiogenesis and fibrosis assay respectively. Sexual satisfaction after the injection of APRFG was clinically difference and the entire patient had sexual satisfaction. In the patient's follow-up, none of them needs to repeat the treatment. Our results declared that APRGF injection was effective and symptoms were disappeared in the entire patients. Significant objective improvements in vaginal diameter (mean before injection, $6.5 \mathrm{~cm} \mathrm{vs} 7.1 \mathrm{~cm}$ after injection) $(\mathrm{p}$-value $=0.001)$ and vaginal flexibility (mean before treatment, $0.72 \mathrm{~cm}$ vs $1.85 \mathrm{~cm}$ after injection) $(\mathrm{P}$-value $=0.026)$ were observed. Characteristics of discharge before the injection in $60 \%$ of patients were included dry vagina and $40 \%$ had mild discharge but after injection $40 \%$ of patients had moderate and also $60 \%$ had mild and sufficient discharge $(\mathrm{P}$-value $=0.190)$. Overally, our patients reported better sexual function and showed better vaginal function indexes, after APRFG injection.
\end{abstract}

Keywords: Autologous- platelet- growth factor- sexual dysfunction- pelvic irradiation

Asian Pac J Cancer Prev, 20 (3), 817-823

\section{Introduction}

Sexual dysfunction is a common sequel to cancer treatment, affecting the quality of life (QOL) in women treated with pelvic radiotherapy (Jensen et al., 2003; Andreyev, 2007; Dunberger et al., 2010; Incrocci and Jensen, 2013). Pelvic radiation may be delivered as the external beam and/or brachytherapy. The accumulated radiation dose to the pelvic organs is important for acute bowel, bladder, and genital toxicity (Jensen and Froeding, 2015). The occurrence of complications is dose and fractionation dependent. Radiation effects are progressive and may become symptomatic after a latent period, but there may be a continuous progression from acute edema, mucosal and sub-mucosal inflammation and persistent ulceration and necrosis to fibrosis. On the longer term, vaginal wall thinning, adhesions atrophy, and fibrosis may occur often followed by decreased vaginal elasticity, narrowing, shortening and ultimately total vaginal stenosis (Schover et al., 1989; Bergmark et al., 1999). A healthy sexual response is described having four phases; desire, excitement, orgasm, and resolution, whereas female sexual dysfunction (FSD), includes desire, arousal, orgasmic and sexual pain disorders (Basson et al., 2000). Dyspareunia (pain during sex) is the most usual ailment in these patients. it's often linked to alterations in the vaginal tissues (stenosis, vaginal fibrosis or atrophy), vaginal size (total vaginal length, genital hiatus) or vaginal dryness which results from in loss of adequate lubrication during intercourse. These changes manifest as superficial or deep dyspareunia. Current studies have evaluated various treatment choices for female dyspareunia and have

${ }^{1}$ Department of Gynecology and Obstetrics, Shariati Hospital, ${ }^{2}$ Hematology, Oncology and Stem Cell Transplantation Research Center, Tehran University of Medical Sciences, ${ }^{3}$ Cell Therapy and Hematopoietic Stem Cell Transplantation Research Center, ${ }^{4}$ Department of Pathology, Shahid Beheshti University of Medical Sciences, Tehran, Iran. *ForCorrespondence: smohammadi@sina.tums.ac.ir, saeedm_58@yahoo.com 
recommended hormone replacement therapy, the use of vaginal dilators and also surgery.

The cited treatment strategies have not been successful, because of the concern around the risk of fistula after surgery and cancer recurrence following estrogen intake (Phillips, 2000; Kingsberg et al., 2010). Therapeutic alternatives for solving these patients are very special because of the median age of adult females who became radiation-induced cancer was so high and the tone of sex life was not a priority for patients (Carter et al., 2017). During the past decade by changing the shape of cancer outbreaks in younger women and progress in developing new radiation methods for cancer treatment, life expectancy has increased and quality of sexual relations has been spotlighted. Over the last decades, the role of platelet-derived growth factors has been highlighted in regenerative medicine (Mostafaei et al.). Plateletderived growth factors are biologic active compounds and also acting in different mechanisms and pathways including activation or induction of chemotaxis, cellular proliferation and angiogenesis (Mohammadi et al., 2016; Mohammadi et al., 2017b). Platelet related products have been a satisfactory outcome in the treatment of chronic skin and soft tissue lesions and plastic surgeries (SerraMestre et al., 2014; Mohammadi et al., 2017a). In new strategies for treatment of FSD, following the cited facets should be considered (Farmer et al., 2016; Carter et al., 2017)

- Do not contain hormonal combinations

- Able to reduce fibrosis and enhances vaginal flexibility

- Can stimulate vaginal secretion and natural lubrication by angiogenesis

- Had a good price

We focused for the first time to identify, describe, and evaluate the safety, symptom resolution and objective improvement the injection of APRGF for treatment of FSD in patients after pelvic irradiation.

\section{Materials and Methods}

\section{Patients and methods}

Demographics data

Ten patients with a history of the dyspareunia after radiation were recruited, in the Tehran University of Medical Sciences, Shariati hospital from July 2017 to December 2017. The age of these patients ranged from 30 years to 56 years. Patients were selected from women who had a history of radiotherapy due to cancer (bladder, uterine, cervix or rectum) with vaginal problems. A summary of patients' characteristics was presented in Table-1.

\section{Patient selection}

This prospective pilot, single arm clinical trial enrolled 10 cancer patients with sexual dysfunction who underwent pelvic radiotherapy at least 5 years ago, randomly. All patients were cancer-free according to according to their clinical and para-clinical evaluation. An exclusion criteria were defined as follows: lower platelet count of $<10^{3} \mu \mathrm{L}$, hemoglobin $<10 \mathrm{~g} / \mathrm{dL}$, anti-proliferative therapies such as chemotherapy in the past 3 months, anti-inflammation medications in the past 2 weeks, radiation in the past 3 months, growth factor therapies in the last 2 weeks, history of diabetes, history of mal-absorption (celiac disease) and any contra-indication in peripheral venues access (Mohammadi et al., 2017b). All the patients were asked to sign a written consent form freely after explanation of all the aspects of study according to their level of knowledge. The cases were referred to the Medical Ethics Committee in order to avoid any conflict of interest. The current study was approved by Tehran University Medical Ethics Committee (IR.TUMS.VCR.REC.1397-4915) and with the Helsinki Declaration of 1975, revised in 1983.

\section{Patients' history and medical data}

Pap smear, abdominal and pelvic CT scan and complete examination are done for all of the patients. Vaginal examination was performed during the screening on those who fulfilled the inclusion and exclusion criteria. The vaginal exam includes a POP-Q, speculum and manual, to evaluate the labia, genital skin, vaginal mucosa, cervix, uterine size (if not surgically removed); mass perception or touch, vaginal flexibility, stenosis, and atrophy was performed. The vaginal flexibility was measured by speculum examination and width of opening blades.

In two patients, we were not capable to place a speculum because of severe stenosis and zero flexibility reported. In three patients, we could just go into the speculum and there was no possibility of opening the blades, we covered the flexibility 0.5 in these patients. Another item that was evaluated at the beginning of the study and follow-up visits was the TVL, which was measured during the POP-Q examination with a graded swab; because shortness of TVL due to radiotherapy fibrosis causes deep dyspareunia. Patient report of vaginal discharge was one of the important points that asked about its quality and quantity in every visit. A natural vaginal discharge is a clear, odorless liquid that facilitates penetration and reduces dyspareunia, by creating lubrication. Its origin of transudate vaginal capillaries is a sign of vaginal refreshment and youth. Vaginal dryness is one of the significant causes of dyspareunia in patients due to lack of hormonal support and fibrosis. This component was evaluated qualitatively through a patient's history and during the examination. It was reported to be severe dryness (never), mild to moderate and satisfactory (adequate) discharge. Intercourse frequency and patient report of sexual satisfaction were evaluated through biographies and special questionnaires at each visit, and the score was recorded. All details were documented and assessed at the end of the survey. Finally, in patients who were satisfied with the sampling, one biopsy of the lateral vaginal wall $(0.5 * 0.5 \mathrm{~cm})$ was taken to compare before and after treatment.

\section{Study protocol and PRP preparation}

Each patient received 1-2 cc PRFG within four weeks (4 times injection) period. For APRGF preparation, first 15 $\mathrm{ml}$ peripheral blood was drawn from a donor using a 20 $\mathrm{mL}$ syringe containing $1.5 \mathrm{ml}$ anticoagulant: sodium citrate 
(ACD-A1) ( Terumo BCT, Inc., USA). Then, the blood was shaken gently 5 times. Afterward, it was transferred into two $10 \mathrm{~mL}$ tubes (Standard kit) (Noavaran Salamat Arzhang, Inc., Iran) and centrifuged at $150 \times \mathrm{g}$ for $10 \mathrm{~min}$ at $24^{\circ} \mathrm{C}$. After first centrifugation, the 2 fold rich platelet in the supernatant plasma was achieved. This platelet-rich plasma (PRP) was then transferred to the second tube for $340 \mathrm{~g}$ (5min) centrifugation. APRGF was obtained from concentrated PRP by freeze and thaw method. Obtained products were centrifuged three times at $900 \mathrm{~g}$ for 30 min to remove platelet bodies (Mohammadi et al., 2016). Finally autologous APRGF injected sub-dermally and/ or intra-muscularly, infiltrating the areas involved of the vaginal walls (eg. Anterior vaginal wall into a space between the vagina and urethra, into the clitoris and lateral vaginal walls), Then the patients were compared for changes in color and appearance of the vaginal tissue and vulva, vaginal discharge and dryness, vaginal flexibility, trans vaginal length, intercourse frequency and sexual satisfaction to determine the need to repeat the treatment.

\section{Lateral wall vaginal biopsy}

According to the signed consent forms taken from the patients, a lateral wall vaginal biopsy must have been done before starting APRGF injection and after final injection in order to evaluate the histological differences. The tissue was evaluated for neovascularization using immunohistochemistry (IHC) assay of CD34+ (BD PharmingenTM, purified rat anti-CD34) positive cells in order to evaluate the angiogenesis. Additionally, Masson's trichrome staining was ordered to investigate the fibrosis before and after treatment.

\section{Treatment assessment}

All ten patients were re-evaluated at eight weeks and six months later to rule out vulvar carcinoma, relapse of the symptom and the need for re-injection.

\section{Statistical Methods}

All data were analyzed using Statistical Package for Social Sciences, SPSS, version 18.0 (SPSS, Inc., Chicago, IL, USA). Continuous variables are presented as mean \pm standard deviation and categorical variables are presented as $\mathrm{N}(\%)$. For comparing the central tendency (e.g. mean for normal and median for non-normal gene expression) between before and after intervention, paired t-test or Wilcoxon signed rank non-parametric test was performed. Any P-value less than 0.05 considered as statistically significant.

\section{Results}

\section{Patients'data}

In this study data were obtained from ten Iranian women aged 30 to 56 years old (mean $=46$ years old), regarding parity (nulliparous 30\%, multiparous $70 \%$ ), sexual activity and frequency of sexual relations (50 $\%$ inactive , 50\% Rare), type of cancer (rectum 20\%, cervix $40 \%$, bladder $20 \%$ and endometrial cancer $20 \%$ ), radiation type (brachytherapy $40 \%$, external radiotherapy $60 \%$ ), history of hormone replacement therapy ( $40 \%$ of
Table 1. Summarizes Patients' Characteristics

\begin{tabular}{lc}
\hline Age (year) & \\
Mean & 46 \\
Medium & 44 \\
Minimum & 30 \\
Maximum & 56 \\
Cancer Type \% (Number) & \\
Rectum cancer & $20 \%(3)$ \\
Uterine cancer & $20 \%(2)$ \\
Bladder cancer & $20 \%(2)$ \\
Cervical cancer & $40 \%(3)$ \\
Radiation Type \% (Number) & \\
External radiotherapy & $60 \%(6)$ \\
Brachytherapy & $40 \%(4)$ \\
Sexual activity \% (Number) & \\
Nulliparous & $30 \%(3)$ \\
Multiparous & $70 \%(7)$ \\
Sexual frequency \% (Number) & \\
Inactive* & \\
Rare** & $50 \%(5)$ \\
History of HRT \% (Number) & $50 \%(5)$ \\
Yes & \\
No & $40 \%(4)$ \\
\hline
\end{tabular}

Activity and frequency of sexual relations: *Inactive: Not having sexual intercourse in the last year, **Rare: Less than 4 times a year

the patients received the HRT in the past and $60 \%$ don't history give HRT). A summary of patients' characteristics was presented in Table 1.

APRGF reduces fibrosis and improvement vaginal flexibility, through increasing the diameter of genital hiatus and vaginal length.

Data showed that symptoms had disappeared in all of the patients with significant objective improvements in vaginal diameter (mean of TVL before PRFG injection was $6.5 \mathrm{~cm}$ vs $7.1 \mathrm{~cm}$ after PRFG injection), p-value $=0.001$. Vaginal flexibility (mean of flexibility before injection was $0.72 \mathrm{~cm}$ vs $1.85 \mathrm{~cm}$ after injection), $\mathrm{P}$-value $=0.026$, showed significant improvement after APRGF injection. Figure 1 and the summarized results in Table 2 were declared that PRGF reduces fibrosis and improvement vaginal flexibility, through increasing the diameter of genital hiatus and vaginal length.

Intercourse frequency and sexual satisfaction improvement after APRGF injection is related to vaginal discharge

Form discharge points of view, $60 \%$ of patients had a dry vagina and $40 \%$ had mild discharge that did not provide the effective lubricant during sexual intercourse. After APRGF injection, $40 \%$ of patients had mild to moderate and $60 \%$ had adequate discharge $(\mathrm{P}$-value $=0.190)$. The color and appearance of the vagina were significantly different before and after treatment. Patient's intercourse frequency after APRGF injection increased and sexual satisfaction improved after treatment. Before study, patients were disagreeing while after APRGF injection $60 \%$ strongly agreed and the entire patients had sexual 

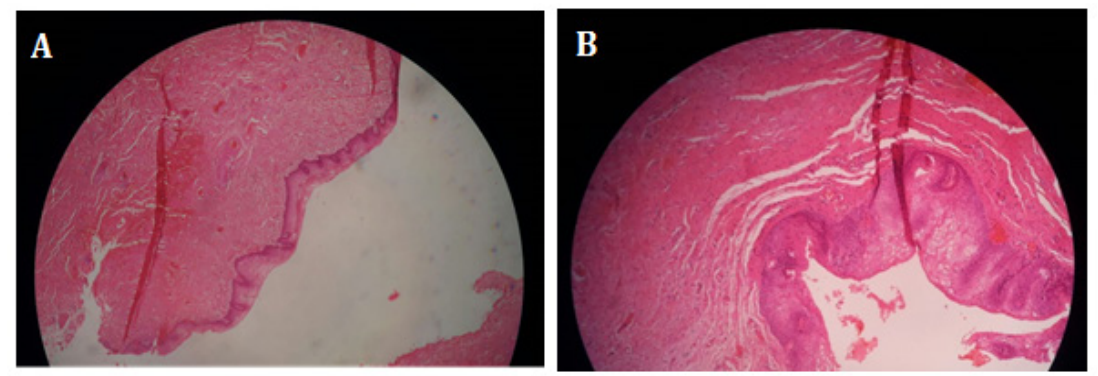

Figure 1. A: Lateral wall vaginal biopsy stained with hematoxylin \& Eosin in 35 years women with rectum cancer that she has not able to intercourse over the last three years (after irradiation) due to severe vaginal atrophy and stenosis. B: The pathology of the same patient after receiving four doses of the ARPGF, with obvious changes in reduction of fibrosis and also angiogenesis.

Table 2. Comparison of Trans Vaginal Length and Vaginal Flexibility, with Speculum before and after Treatment

\begin{tabular}{lcccc}
\hline & $\begin{array}{c}\text { TVL before } \\
\text { treatment }\end{array}$ & $\begin{array}{c}\text { TVL after } \\
\text { treatment }\end{array}$ & $\begin{array}{c}\text { Vaginal flexibility } \\
\text { before treatment }\end{array}$ & $\begin{array}{c}\text { Vaginal flexibility } \\
\text { after treatment }\end{array}$ \\
\hline Number & 10 & & & 10 \\
$\quad$ Valid & 0 & 10 & 10 & 0 \\
$\quad$ Missing & 6.55 & 0 & 0 & 1.85 \\
Mean & 6.2 & 7.1 & 0.72 & 1.75 \\
Median & 0.96177 & 7 & 0.6 & 0.66875 \\
Std. Deviation & 0.85 & 0.85375 & 0.53292 & 0.334 \\
Skewness & 0.687 & 0.359 & 0.197 & 0.687 \\
Std. Error of Skewness & -0.652 & 0.687 & 0.687 & -0.852 \\
Kurtosis & 1.334 & -1.196 & -0.829 & 1.334 \\
Std. Error of Kurtosis & 5.5 & 1.334 & 1.334 & 1 \\
Minimum & 8.2 & 6 & 0 & 3 \\
Maximum & & 8.5 & 1.5 & 1.375 \\
Percentiles & 5.9 & & & 1.75 \\
25 & 6.2 & 6.35 & 0.375 & 2.5 \\
50 & 7.475 & 7 & 0.6 & 1.125 \\
75 & & 8 & & \\
\hline
\end{tabular}

satisfaction which was related to TVL, vaginal flexibility and discharge. During the follow-up visit, any patient needs to repeat the treatment. Patient report of vaginal discharge and sexual satisfaction before and after treatment with PRGF were summarized in Table 3.

\section{Histopathological assessment}

Comparison of vaginal wall biopsy showed improvement of atrophy, fibrosis, and angiogenesis after PRGF injection (Figure 1). The reticulin staining demonstrated a significant decrease in fibrosis after PRGF injection and also showed subepithelial loose fibroconnective tissue without fibrosis (Figure 2A). The micro-vessels formation (angiogenesis) was evaluated in all the patients through a lateral vaginal biopsy. There was no significant difference before and after APRGF injection in IHC staining for CD34 in this biopsy section after four weeks (4 doses injection) (Figure 2B).

\section{Discussion}

Sexual dysfunction is a common sequel to cancer treatment, in women treated with pelvic radiotherapy. This complication increased over the time and was greater for cancer patients who were female, older, married or in a committed relationship (Acquati et al., 2017). The cohort

Table 3. Patient Report of Vaginal Discharge and Sexual Satisfaction before and after Injection of APRGF

\begin{tabular}{|c|c|c|c|c|c|c|}
\hline \multirow[b]{2}{*}{ Before treatment } & \multicolumn{2}{|c|}{ Intercourse frequency } & \multicolumn{2}{|c|}{ Patient report of vaginal discharge } & \multicolumn{2}{|c|}{ Sexual satisfaction } \\
\hline & Inactive & $50 \%$ & Dry & $60 \%$ & Disagree & $50 \%$ \\
\hline & Rare & $50 \%$ & Mild & $40 \%$ & Agree a little & $50 \%$ \\
\hline & Active & $0 \%$ & Adequate & $0 \%$ & Strongly agree & $0 \%$ \\
\hline \multirow[t]{3}{*}{ After treatment } & Inactive & $0 \%$ & Dry & $0 \%$ & Disagree & $10 \%$ \\
\hline & Rare & $30 \%$ & Mild & $40 \%$ & Agree a little & $40 \%$ \\
\hline & Active & $70 \%$ & Adequate & $60 \%$ & Strongly agree & $50 \%$ \\
\hline
\end{tabular}




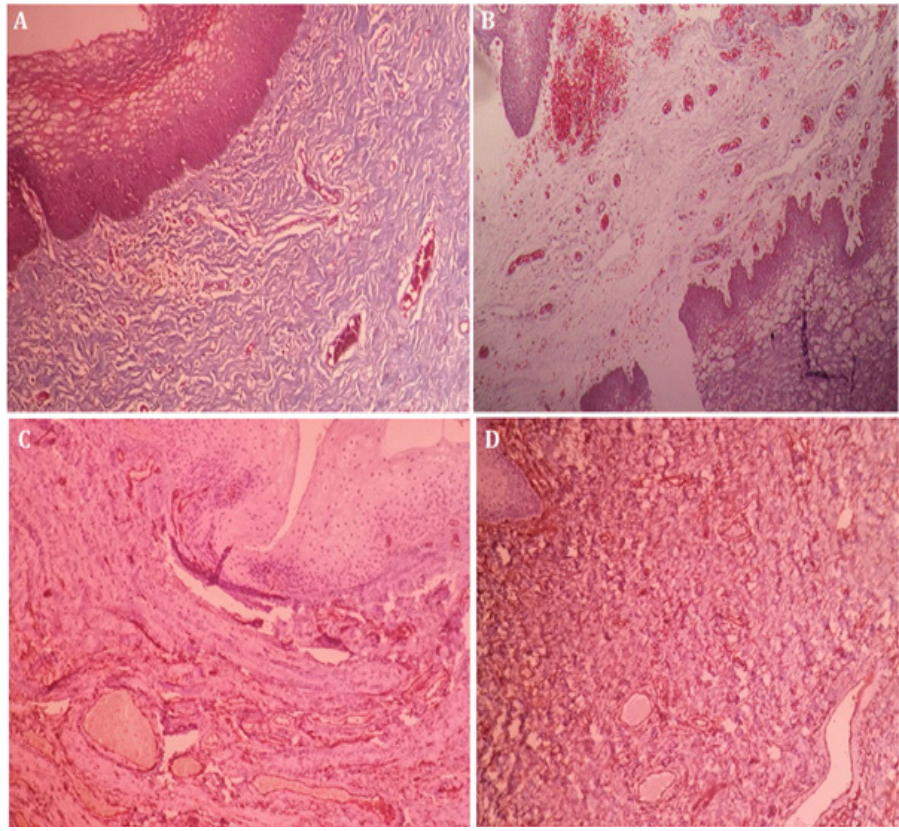

Figure 2. Lateral Wall Vaginal Biopsy Stained with Masson's Trichrome Staining and IHC CD34) for Evaluation of Angiogenesis in 35 Years Women with Rectum Cancer that She has not Able to Intercourse Over the Last Three Years (after irradiation) due to Severe Vaginal Atrophy and Stenosis. A and C are belonged to this patient before receiving APRGF and $B$ and $D$ are belonged to the same patient after receiving three doses of the ARPGF. A: showed subepithelial dense fibrosis $(40 \times)$. B: Subepithelial loose fibroconnective tissue without fibrosis $(40 \times)$. C and D: there is no significant difference before and after APRGF injection in IHC staining for CD34 in this biopsy section (40×).

profile was designed for the first time by the Women's Health Clinic (WHC) at Mayo Clinic in Rochester, Minnesota, medical information. They were evaluated 8688 women from 2005 to 2017 , to determine the prevalence of the sexual dysfunction and its relationship with health, disease, age and so on. The study showed a high need for curative and preventive sexological care to improve quality of life of women with infertility (Faubion et al., 2018). Available therapeutic options such as vaginoplasty and hormone replacement therapy are not suitable. The risk of fistula and secondary reconstruction vaginal stenosis following surgery and a relapse of the tumor due to hormonal administration are the common concern of these therapeutic strategies (Kingsberg et al., 2010; Carter et al., 2017). Owing to the many probable etiologic factors involved in FSD and the inconsistency in the response to current treatment modalities, this part needs research to introduce effective therapeutic options (Buster, 2013; Milbury et al., 2013).

In new strategies for treatment of FSD, ability in fibrosis reduction enhances vaginal flexibility and stimulation of vaginal secretion via angiogenesis induction should be considered (Farmer et al., 2016). Based on the ability of PRP in the induction of angiogenesis and tissue regeneration, it seems to be a suitable choice in this area (Mostafaei et al.; Mohammadi et al., 2017a; Mohammadi et al., 2017b). Thus, the effort to use lowcomplicated treatments that could rejuvenate the patient to pre-radiotherapy conditions led us to use APRGF. The result of the present study for the first time reported better sexual and vaginal function indexes after APRFG injection in patients undergoing pelvic irradiation. Our histopathology data showed that injection of APRGF into the anatomic regions involved in sexual responsiveness via growth factors and cytokines might promote differentiation of pluripotent stem cells and induction of neoangiogenesis, glandular proliferation (Skene's glands), fibrosis reduction and new neuronal growth. These changes eventually could be improved physiologic responsiveness. Hence, we presumed that amended vascularity and neuronal regrowth in the vagina and in the clitoral area could augment sexual responsiveness through enhancing blood flow to the area, particularly in cases where hormonally independent vaginal atrophy contributes to FSD. In addition to increased blood flow, collagen and sensory nerve regrowth might relieve coital discomfort as well as enhance vaginal sensitivity. Also, increased blood flow in the clitoris, if induced by PRP injections, could also lead to improved arousal and orgasm. Our recent studies demonstrated that PRP induces regrowth of new tissue through activation of pluripotent stem cells and also angiogenesis in average 5 weeks after injection. These cells are capable of differentiating into several tissue types when stimulated by growth factors produced by activated platelets. (Mostafaei et al.; Mohammadi et al., 2017a; Mohammadi et al., 2017b). Platelets contained granules and growth factors, such as TGF- $\beta$, PDGF, IGF, VEGF, EGF and FGF-2 (Christgau et al., 2006), which stimulate the inflammatory cascade and healing process. PDGF plays an important role in cell proliferation, angiogenesis tissue and mucous regeneration (Kim and Yun, 2007; Alio et al., 2012; Pal et al., 2012; Sclafani and McCormick, 2012). Angiogenesis can be effective in the production of natural vaginal discharge and lubrication during intercourse. PDGF is a required element in the cellular division for fibroblasts, a type of connective tissue cell that is the most effective factor in reducing vaginal fibrosis and stenosis and increasing 
vaginal flexibility (Song et al., 2005; Anitua et al., 2009). Since based on our previous study angiogenesis need more than 4 weeks, we hypothesized that this no significant differences in CD34 IHC might be related to the time of taking biopsy (Mohammadi et al., 2017b). In line with our findings, Charles Runels and colleagues in their pilot study demonstrated that specifically placed intra-vaginal and intra-clitoral PRP injections could be an effective method to treat certain types of FSD, especially in the areas of desire, arousal, lubrication, and orgasm. In spite of our findings, improvement in satisfaction and pain were not statistically significant in their study (Runels, 2014).

The result of Behnia et al; showed that PRP presents a potential alternative to topical steroids for treatment of vulvovaginal autoimmune conditions such as lichen sclerosus. They hypothesized growth factors released by platelets, monocytes, and nutrients have an important role in phagocytosis of fibrotic tissue, inflammation reduction, angiogenesis stimulation, and collagen III synthesis (Behnia-Willison et al., 2016).

In conclusion, to sum up, in this study, ten patients had dyspareunia and extraordinarily vaginal stenosis and dryness mucous were treated with the APRGF as prescribed. Our patients reported better sexual function and showed better vaginal function indexes, after APRFG injection. However results were positive but mandate further investigation due to limitation of our small pilot study,

\section{Conflicts of Interest}

The authors report no conflicts of interest.

\section{Acknowledgments}

We would like to thank department of Gynecology\& Obstetrics, Shariati Hospital and also Hematology Oncology and Stem Cell Transplantation Research Center, Tehran University of Medical Sciences. Furthermore, special thanks for Noavaran Salamat Arzhang in order to support this study.

\section{References}

Acquati C, Zebrack BJ, Faul AC, et al (2017). Sexual functioning among young adult cancer patients: A 2-year longitudinal study. Cancer, DOI: 10.1002/cncr.31030.

Alio JL, Arnalich-Montiel F, Rodriguez AE (2012). The role of "eye platelet rich plasma" (E-PRP) for wound healing in ophthalmology. Curr Pharm Biotechnol, 13, 1257-65.

Andreyev HJ (2007). Gastrointestinal problems after pelvic radiotherapy: the past, the present and the future. Clin Oncol (R Coll Radiol), 19, 790-9.

Anitua E, Sanchez M, Zalduendo MM, et al (2009). Fibroblastic response to treatment with different preparations rich in growth factors. Cell Prolif, 42, 162-70.

Basson R, Berman J, Burnett A, et al (2000). Report of the international consensus development conference on female sexual dysfunction: definitions and classifications. J Urol, 163, 888-93.

Behnia-Willison F, Pour NR, Mohamadi B, et al (2016). Use of platelet-rich plasma for vulvovaginal autoimmune conditions like lichen sclerosus. Plast Reconstr Surg Glob Open, 4, e1124.
Bergmark K, Avall-Lundqvist E, Dickman PW, et al (1999). Vaginal changes and sexuality in women with a history of cervical cancer. $N$ Engl J Med, 340, 1383-9.

Buster JE (2013). Managing female sexual dysfunction. Fertil Steril, 100, 905-15.

Carter J, Stabile C, Seidel B, et al (2017). Vaginal and sexual health treatment strategies within a female sexual medicine program for cancer patients and survivors. J Cancer Surviv, 11, 274-83.

Christgau M, Moder D, Hiller KA, et al (2006). Growth factors and cytokines in autologous platelet concentrate and their correlation to periodontal regeneration outcomes. J Clin Periodontol, 33, 837-45.

Dunberger G, Lind H, Steineck G, et al (2010). Fecal incontinence affecting quality of life and social functioning among long-term gynecological cancer survivors. Int $J$ Gynecol Cancer, 20, 449-60.

Farmer M, Yoon H, Goldstein I (2016). Future targets for female sexual dysfunction. $J$ Sex Med, 13, 1147-65.

Faubion SS, Kapoor E, Kling JM, et al (2018). Data registry on experiences of aging, menopause, and sexuality (DREAMS): A cohort profile. Maturitas, 107, 44-9.

Incrocci L, Jensen PT (2013). Pelvic radiotherapy and sexual function in men and women. $J$ Sex Med, 10, 53-64.

Jensen PT, Froeding LP (2015). Pelvic radiotherapy and sexual function in women. Transl Androl Urol, 4, 186-205.

Jensen PT, Groenvold M, Klee MC, et al (2003). Longitudinal study of sexual function and vaginal changes after radiotherapy for cervical cancer. Int $J$ Radiat Oncol Biol Phys, 56, 937-49.

Kim TJ, Yun YP (2007). Antiproliferative activity of NQ304, a synthetic 1,4-naphthoquinone, is mediated via the suppressions of the PI3K/Akt and ERK1/2 signaling pathways in PDGF-BB-stimulated vascular smooth muscle cells. Vascul Pharmacol, 46, 43-51.

Kingsberg S, Kellogg S, Krychman M (2010). Treating dyspareunia caused by vaginal atrophy: a review of treatment options using vaginal estrogen therapy. Int $J$ Womens Health, 1, 105-11.

Milbury K, Cohen L, Jenkins R, et al (2013). The association between psychosocial and medical factors with long-term sexual dysfunction after treatment for colorectal cancer. Support Care Cancer, 21, 793-802.

Mohammadi MH, Molavi B, Mohammadi S, et al (2017a). Evaluation of wound healing in diabetic foot ulcer using platelet-rich plasma gel: A single-arm clinical trial. Transfus Apher Sci, 56, 160-4.

Mohammadi S, Nasiri S, Mohammadi MH, et al (2017b). Evaluation of platelet-rich plasma gel potential in acceleration of wound healing duration in patients underwent pilonidal sinus surgery: A randomized controlled parallel clinical trial. Transfus Apher Sci, 56, 226-32.

Mohammadi S, Nikbakht M, Malek Mohammadi A, et al (2016). Human platelet lysate as a xeno free alternative of fetal bovine serum for the in vitro expansion of human mesenchymal stromal cells. Int J Hematol Oncol Stem Cell Res, 10, 161-71.

Mostafaei S, Norooznezhad F, Mohammadi S, Norooznezhad AH (2017). Effectiveness of Platelet-rich Plasma Therapy in Wound Healing of Pilonidal Sinus Surgery: A Comprehensive Systematic Review and Meta-analysis. Wound Repair and Regeneration, 25, 1002-7.

Pal US, Mohammad S, Singh RK, et al (2012). Platelet-rich growth factor in oral and maxillofacial surgery. Natl $J$ Maxillofac Surg, 3, 118-23.

Phillips NA (2000). Female sexual dysfunction: evaluation and treatment. Am Fam Physician, 62, 127-36, 41-2. 
Runels C (2014). A pilot study of the effect of localized injections of autologous platelet rich plasma (PRP) for the treatment of female sexual dysfunction. Womens Health Care, 3, 2-4.

Schover LR, Fife M, Gershenson DM (1989). Sexual dysfunction and treatment for early stage cervical cancer. Cancer, $\mathbf{6 3}$, 204-12.

Sclafani AP, McCormick SA (2012). Induction of dermal collagenesis, angiogenesis, and adipogenesis in human skin by injection of platelet-rich fibrin matrix. Arch Facial Plast Surg, 14, 132-6.

Serra-Mestre JM, Serra-Renom JM, Martinez L, et al (2014). Platelet-rich plasma mixed-fat grafting: a reasonable prosurvival strategy for fat grafts?. Aesthetic Plast Surg, 38, 1041-9.

Song G, Ouyang G, Bao S (2005). The activation of Akt/PKB signaling pathway and cell survival. J Cell Mol Med, 9, $59-71$.

\section{cc) (i) (3)}

This work is licensed under a Creative Commons AttributionNon Commercial 4.0 International License. 